\title{
Effect of smear layer characteristics on dentin bonding durability of HEMA-free and HEMA-containing one-step self-etch adhesives
}

\author{
Yuko SHINODA ${ }^{1}$, Masatoshi NAKAJIMA ${ }^{1}$, Keiichi HOSAKA ${ }^{1}$, Masayuki OTSUKI ${ }^{1}$, Richard M. FOXTON² \\ and Junji TAGAMI ${ }^{1,3}$
}

\begin{abstract}
${ }^{1}$ Cariology and Operative Dentistry, Department of Restorative Sciences, Graduate School of Medical and Dental Sciences, Tokyo Medical and Dental University, 1-5-45 Yushima, Bunkyo-ku, Tokyo 113-8549, Japan

${ }^{2}$ Department of Conservative Dentistry, King's College London Dental Institute at Guy's, King's and St Thomas' Hospitals, King's College London, London Bridge, London, SE1-9RT, UK

${ }^{3}$ Global Center of Excellence Program; International Research Center for Molecular Science in Tooth and Bone Diseases at Tokyo Medical and Dental University, 1-5-45 Yushima, Bunkyo-ku, Tokyo 113-8549, Japan
\end{abstract}

Corresponding author, Masatoshi NAKAJIMA; E-mail: nakajima.ope@tmd.ac.jp

\begin{abstract}
The purpose of this study was to evaluate the effect of smear layer characteristics on the dentin bonding durability of HEMA-free and HEMA-containing one-step self-etch adhesives. Xeno V (XV; HEMA-free), G BOND PLUS (GB; HEMA-free) and Clearfil $\mathrm{S}^{3}$ Bond (S${ }^{3}$; HEMA-containing), were applied to dentin surfaces prepared with either \#180- or \#600-grit SiC paper according to manufacturers' instructions. Bond strengths to dentin were determined using $\mu$ TBS test after 24-hour, 6-month, and 1-year water storage. In addition, nanoleakage evaluation was performed using an SEM. The smear layer characteristics affected water-tree nanoleakage formation in the adhesive layers of XV and GB, which contributed to a reduction in $\mu$ TBS after 6-month water storage, while the characteristics did not affect the $\mu \mathrm{TBS}$ of $\mathrm{S}^{3}$. However, regardless of the smear layer characteristics, 1-year water storage significantly reduced the $\mu$ TBS of all the adhesives and was associated with an increase in failures at the adhesive-composite interface.
\end{abstract}

Keywords: One-step self-etch adhesives, Dentin bonding durability, Smear layer characteristics, Nanoleakage, HEMA

\section{INTRODUCTION}

Bonding durability is an important factor in the maintenance of the integrity of adhesive restorations. It has been demonstrated that discrepancies between the depth of demineralization and resin infiltration would create three-dimensional leakage pathways within the hybrid layer, which has been called nanoleakage ${ }^{1)}$. Nanoleakage permits water penetration which may accelerate plasticization of the adhesive resin and hydrolysis of exposed collagen within the hybrid layer, resulting in degradation of the hybrid layer over time $e^{2,3)}$. Self-etch adhesives are likely to show less nanoleakage because of simultaneous demineralization and resin infiltration into the dentin substrate. However, the previous study using two-step self-etch adhesives demonstrated that nanoleakage gradually increased within the hybrid layer after long-term water storage, leading to a reduction in dentin bond strength and specimen failures within the hybrid layer ${ }^{4)}$.

Recently, one-step self-etch adhesives have been developed to simplify bonding procedures, which combine the three bonding steps of etching, priming, and bonding into a single step. It has been reported that current one-step self-etch adhesives showed good initial bond strengths to dentin ${ }^{5,6)}$, however, there are still many concerns regarding their bonding durability. Recent studies have shown that the bond strengths of one-step self-etch adhesives to dentin reduced after long-term water storage ${ }^{7-10)}$, with an associated increase in the number of cohesive failures in adhesive resin, and a reduction in the number of interfacial failures ${ }^{8,10)}$. These results would indicate that the long-term reduction in bond strength is due to degradation of the adhesive resin layer. One-step self-etch adhesives have higher hydrophilicity after polymerization, causing higher water sorption ${ }^{11-14)}$, and they act as permeable membranes that permit water movement across their thickness ${ }^{15-17)}$. The absorbed water is known to reduce the mechanical properties of the resin polymers ${ }^{11,18,19)}$. Increasing water sorption might accelerate a reduction in the mechanical properties of one-step self-etch adhesives, leading to compromised dentin bonding durability. The water sorption in one-step self-etch adhesives is dependent upon their HEMA concentration ${ }^{14,19)}$. Therefore, HEMA-free one-step self-etch adhesive systems have recently been introduced with the expectation of reducing water sorption and increasing the long-term stability of the mechanical properties. Ultrastructural observation of the adhesive interface of one-step self-etch adhesives has shown nanoleakage in the adhesive layer as well as in the hybrid layer ${ }^{20,21)}$. Additionally, HEMA-free adhesives express water-tree nanoleakage formation above the resin-dentin interface due to phase separation of adhesive components and osmotic water infiltration as a result of water remaining on the dentin substrate or being transported from the dentinal tubules ${ }^{15,22,23)}$. However, there is less research on the effects of water-tree nanoleakage on the dentin bonding durability of HEMA-free adhesives.

The characteristics of the dentin smear layer are known to affect the quality of the hybrid layer of self-etch 
adhesives ${ }^{24,25)}$. The thickness, roughness and density of the smear layer vary with the coarseness level of abrasive papers and burs used ${ }^{26}$. For self-etch adhesives, the smear layer would cause the early neutralization of the adhesive by the buffering components in the dentin smear layer and hamper superficial demineralization of solid dentin ${ }^{26}$. As a result, the smear layer is regarded as a natural barrier for infiltration of adhesive monomer, and affects formation of the hybrid layer. Moreover, the smear layer may influence water-tree formation in the adhesive layer, because the water content of the smear layer and/or water diffusion from the dentinal tubules would be dependent upon the smear layer characteristics with sub-micron porosities ${ }^{27}$. To date, the effect of smear layer characteristics on the dentin bonding durability of one-step self-etch adhesives has not been well understood. Therefore, the objective of this study was to investigate the effect of dentin smear layer characteristics on the bond strengths of HEMA-free and HEMA-containing one-step self-etch adhesives after 24 hours, 6 months and 1 year of water storage, and also to compare dentin bonding durability between HEMA-free and HEMAcontaining adhesives. The null hypothesis to be tested was that neither the smear layer characteristics nor the presence of HEMA in adhesives affect the initial or long-term bond strengths of one-step self-etch adhesives to dentin.

\section{MATERIALS AND METHODS}

Two commercially available HEMA-free one-step self-etch adhesives, Xeno V (XV; Dentsply De Trey, Konstanz, Germany), G BOND PLUS (GB; GC Corp., Tokyo, Japan) and one HEMA-containing one-step self-etch adhesive, Clearfil $\mathrm{S}^{3}$ Bond ( $\mathrm{S}^{3}$; Kuraray Medical Inc., Tokyo, Japan) were used in this study.

\section{Micro-tensile bond strength test}

The method of specimen preparation for the microtensile bond strength ( $\mu$ TBS) test is illustrated in Fig. 1. Thirty caries-free extracted human third molars stored frozen, of which the original patients could not be identified, were used in this study, according to a protocol approved by the Human Research Ethics Committee, Tokyo Medical and Dental University, Japan. The occlusal enamel was removed perpendicular to the long axis of the tooth using a model trimmer under running water. Smear layers with different characteristics were prepared on flat dentin surfaces by grinding with either \#180- or \#600-grit SiC paper under running water. Three

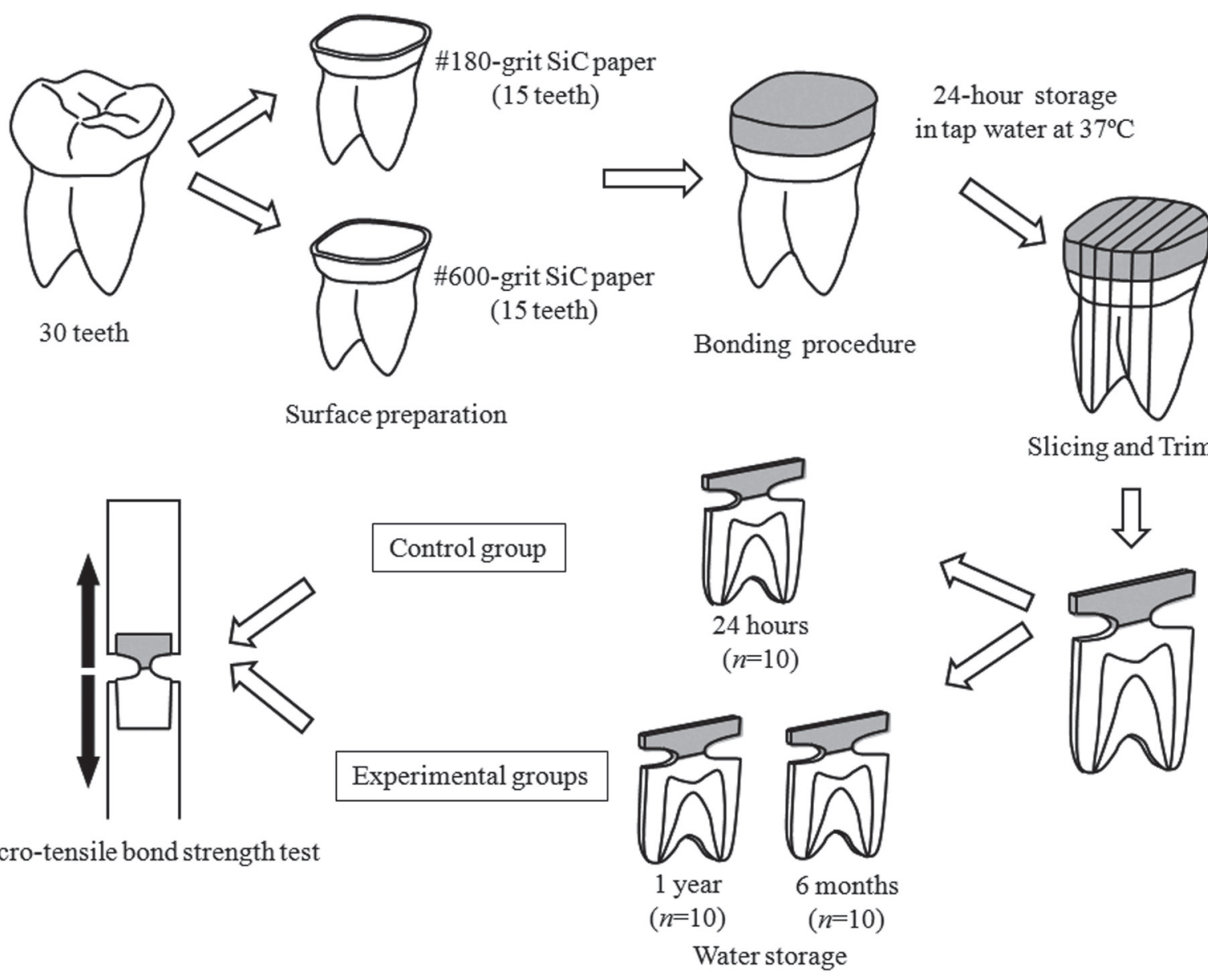

Fig. 1 Schematic illustration of specimen preparation, water storage and micro-tensile bond strength test. 
Table 1 Chemical composition and application mode of the materials tested in this study

\begin{tabular}{lll}
\hline Materials & Composition & Procedures \\
\hline $\begin{array}{l}\text { Xeno V } \\
\text { (XV; Dentsply De Trey) } \\
\text { pH=1.3 }\end{array}$ & $\begin{array}{l}\text { Bifunctional acrylic amides, acrylamido } \\
\text { alkylsulfonic acid, 'inverse' functionalized } \\
\text { phosphoric acid ester, acrylic acid, water, tertiary } \\
\text { butanol, butylated benzenediol, CQ }\end{array}$ & $\begin{array}{l}\text { Apply twice } \\
\text { Agitate for } 20 \mathrm{~s} \\
\text { Dry for } 5 \mathrm{~s} \\
\text { Light cure for } 20 \mathrm{~s}\end{array}$ \\
$\begin{array}{ll}\text { G BOND PLUS } \\
(\mathrm{GB} ; \mathrm{GC})\end{array}$ & $\begin{array}{l}\text { 4-MET, phosphoric acid ester monomer, DMA, } \\
\text { wH=1.5 }\end{array}$ & $\begin{array}{l}\text { Apply for } 10 \mathrm{~s} \\
\text { water, acetone, silica filler, photo-initiator }\end{array}$ \\
$\begin{array}{l}\text { Clearfil } \mathrm{S}^{3} \text { Bond } \\
\left(\mathrm{S}^{3} ; \text { Kuraray Medical) }\right. \\
\text { pH=2.7 }\end{array}$ & $\begin{array}{l}\text { 10-MDP, HEMA, Bis-GMA, water, ethanol, } \\
\text { silanated colloidal silica, CQ }\end{array}$ & Light cure for $10 \mathrm{~s}$ \\
\hline
\end{tabular}

Abbreviations: CQ: camphorquinone; 4-MET: 4-methacryloxyethyl trimellitic acid; DMA: dimethacrylate; 10-MDP:

10-methacryloyloxydecyl dihydrogen phosphate; HEMA: 2-hydroxyethyl methacrylate; Bis-GMA: bisphenol-A-diglycidyl methacrylate

one-step self-etch adhesives were applied to the prepared dentin surfaces according to manufacturers' instructions (Table 1).

After polymerization of the adhesives using a light-curing unit (XL3000, 3M ESPE, St. Paul, MN, USA) with an intensity of $600 \mathrm{~mW} / \mathrm{cm}^{2}$, resin crowns were built up incrementally in three layers with Clearfil AP-X (Kuraray Medical Inc.) to a height of between 4 and $5 \mathrm{~mm}$. Each layer was light-cured for 20 seconds. After storage in tap water at $37^{\circ} \mathrm{C}$ for 24 hours, the bonded teeth were serially sectioned into $0.7 \mathrm{~mm}$-thick slabs using a low speed diamond saw (Isomet, Buehler Ltd., Lake Bluff, IL, USA) under water-cooling. Six slabs per tooth could be harvested for testing. The dentinadhesive interface was trimmed to an hour-glass shape with a $1 \mathrm{~mm}^{2}$ cross-sectional area $(0.7 \times 1.4 \mathrm{~mm})$, using a superfine diamond bur (SF114, Shofu Inc., Kyoto, Japan) mounted in a high-speed turbine handpiece under copious air-water spray. The hour-glass shaped specimens were randomly divided into two experimental groups (6-month and 1-year water storage groups) and a control group (24-hour group). The specimens of the experimental groups were individually stored in tap water at $37^{\circ} \mathrm{C}$ for 6 months or 1 year. The water in which the slabs were stored was changed weekly until testing.

After the designated storage time, the specimen was bonded to a jig using a cyanoacrylate adhesive (Zapit, Dental Ventures of America, CA, USA) mounted in a tabletop testing machine (EZ Test, Shimadzu Co., Kyoto, Japan) and subjected to the $\mu$ TBS test at a crosshead speed of $1.0 \mathrm{~mm} / \mathrm{min}$.

\section{Failure mode analysis}

After the bond strengths were measured, the debonded specimens were mounted on brass stubs, gold sputtercoated and observed using a scanning electron microscopy (SEM) (JSM-5310LV, JEOL, Tokyo, Japan) in order to determine failure mode. The failure modes were classified into four categories: (1) C-D; cohesive failure in dentin, (2) I-F (D-A); interfacial failure at dentin-adhesive interface, (3) $\mathrm{C}-\mathrm{A}$; cohesive failure in adhesive resin, and (4) I-F (A-C); interfacial failure at adhesive-composite interface. I-F (D-A) was defined as regions between the top of hybrid layer and the bottom of hybrid layer. I-F (A-C) was defined if detached or missing filler particles of resin composite were observed between the adhesive resin and resin composite layer. The SEM micrographs were digitized by scanning them into a computer and then subjected to quantitative image analysis using SemAfore software (Insinööritoimisto, J. Rimppi Oy, Finland). The area of each failure mode was calculated on the dentin sides of each debonded specimen and expressed as percentage per overall fractured surface of the specimen.

SEM observation of dentin surfaces treated with one-step self-etch adhesives

Flat dentin disks with a thickness of around $1.0 \mathrm{~mm}$ were prepared from the mid-coronal dentin of six extracted human third molars using a low-speed diamond saw (Isomet). The dentin surfaces were prepared with either \#180- or \#600-grit SiC paper under running water. These surfaces were further treated with the adhesive of $\mathrm{XV}, \mathrm{GB}$ or $\mathrm{S}^{3}$ without light curing, followed by rinsing with $50 \%$ acetone for 5 minutes to remove the adhesives. After rinsing the surfaces with acetone, they were dehydrated in ascending grades of ethanol in the following steps: $25 \%$ for 20 minutes, $50 \%$ for 20 minutes, $75 \%$ for 20 minutes, $95 \%$ for 30 minutes and $100 \%$ for 60 minutes. After the final ethanol step, the dentin disks were dried by immersion in hexamethyldisilazane (HMDS) for 10 minutes, placed on filter paper inside a covered glass vial and air-dried at room temperature for 24 hours $^{28)}$. After drying, the surfaces of the dentin disks were gold-sputter coated and observed using an SEM (JSM-5310LV).

\section{Nanoleakage evaluation}

In order to evaluate nanoleakage at the resin-dentin interface, thirty-six extracted human third molars were 
used. Bonded specimens were prepared in the same manner as the specimen preparation for the $\mu$ TBS test and then these were vertically sectioned into $1.0 \mathrm{~mm}$ thick slices. The two central slabs were chosen from each tooth. All of the slabs were randomly divided into three groups according to storage time (24 hours, 6 months and 1 year). After the designated storage time, the slabs were individually placed in $50 \mathrm{wt} \%$ ammoniacal silver nitrate solution for 24 hours in the dark. Ammoniacal silver nitrate was prepared according to the method described by Tay and others ${ }^{29)}$. After silver staining, the specimens were rinsed thoroughly in distilled water and placed in photo-developing solution for eight hours under a fluorescent light. The specimens were then placed inside an acrylic ring that was attached to double-sided adhesive tape and embedded in epoxy resin. The epoxyembedded specimens were ground flat using $\mathrm{SiC}$ papers up to \#2000-grit and polished with diamond pastes down to $1 / 4 \mu \mathrm{m}$ particle size under running water. They were ultrasonically cleaned and dried for 24 hours at room temperature. After gold-sputter coating, the resin-dentin interface was observed using an SEM (JSM-5310LV).

\section{Statistical analysis}

The statistical analysis was performed using the statistical package for the medical science (SPSS Ver.11 for Windows, SPSS Inc., Chicago, IL, USA). Data from the $\mu$ TBS test and failure mode analysis were initially tested for normality using the Kolmogorov-Smirnov test. The $\mu$ TBS data were analyzed using a three-way ANOVA to examine the effect of the three factors (material, storage time, and preparation method). The data were then analyzed using Dunnett's T3 multiple comparison tests to identify significant differences induced by storage time. Additionally, $t$-tests were used to compare statistical differences between the preparation methods. All analyses were carried out at a 95\% level of confidence. The percentage distributions of each failure mode of the specimens, which were prepared with either \#180- or \#600-grit SiC paper and treated with each adhesive system, were analyzed using Kruskal-Wallis one-way ANOVAs $(p<0.05)$ and Mann-Whitney U-tests combined with the Bonferroni correction $(p<0.016)$.

\section{RESULTS}

\section{Micro-tensile bond strength test}

The micro-tensile bond strength ( $\mu$ TBS) results are shown in Table 2. Three-way ANOVA revealed that all three factors significantly affected the $\mu$ TBS (material; $p<0.001$, storage time; $p<0.001$, preparation method; $p=0.002$ ). In addition, there were significant interactions between two factors in each combination (material vs storage time; $p=0.026$, storage time vs preparation method; $p=0.017$, preparation method vs material; $p<0.001$ ).

For the 24-hour control and 1-year water storage groups, there were no significant differences in $\mu$ TBS of each adhesive between the \#180-grit and \#600-grit SiC paper groups $(p>0.05)$. In contrast, after 6 months of water storage, in the case of the specimens of XV and GB, the \#180-grit SiC paper group showed significantly lower bond strength than the \#600-grit SiC paper group $(p<0.05)$, however there was no significant difference in $\mu$ TBS between the groups of $\mathrm{S}^{3}(p>0.05)$. One year of water storage significantly reduced the $\mu$ TBS of all the tested adhesives in both the \#180-grit and \#600-grit SiC paper groups $(p<0.05)$.

\section{Failure mode analysis}

The percentage distributions of the failure modes are summarized in Fig. 2. For the 24-hour control and 6-month water storage groups in the \#180-grit and \#600-grit SiC paper groups, the main failure mode of all the tested adhesives was cohesive failure in adhesive resin $(\mathrm{C}-\mathrm{A})$. In addition, the percentage of $\mathrm{C}-\mathrm{A}$ of the 6 -month water storage group was higher than that of the 24-hour control group, although there was no significant

Table 2 Micro-tensile bond strength values $(\mathrm{MPa})$ of three one-step self-etch adhesives tested in this study

\begin{tabular}{|c|c|c|c|c|}
\hline \multirow{2}{*}{ Materials } & \multirow{2}{*}{ SiC papers by grit size } & \multicolumn{3}{|c|}{ Time } \\
\hline & & 24 hours & 6 months & 1 year \\
\hline \multirow{3}{*}{$\mathrm{XV}$} & \#180 & $33.9(8.0)^{\mathrm{A}}$ & $29.5(4.8)^{\mathrm{A}}$ & $22.2(6.3)^{\mathrm{B}}$ \\
\hline & & $\mathrm{NS}$ & $p<0.05$ & NS \\
\hline & $\# 600$ & $37.9(7.9)^{\mathrm{A}}$ & $41.9(6.3)^{\mathrm{A}}$ & $26.1(4.0)^{\mathrm{B}}$ \\
\hline \multirow{3}{*}{ GB } & $\# 180$ & $44.0(8.6)^{\mathrm{A}}$ & $38.7(6.8)^{\mathrm{A}}$ & $33.9(3.9)^{\mathrm{B}}$ \\
\hline & & NS & $p<0.05$ & NS \\
\hline & $\# 600$ & $44.3(3.3)^{\mathrm{A}}$ & $47.8(7.7)^{\mathrm{A}}$ & $37.7(4.7)^{\mathrm{B}}$ \\
\hline \multirow{3}{*}{$\mathrm{S}^{3}$} & $\# 180$ & $43.1(3.8)^{\mathrm{A}}$ & $40.0(3.3)^{\mathrm{A} \mathrm{B}}$ & $36.9(3.4)^{\mathrm{B}}$ \\
\hline & & $\mathrm{NS}$ & $\mathrm{NS}$ & $\mathrm{NS}$ \\
\hline & \#600 & $40.1(3.2)^{\mathrm{A}}$ & $36.6(4.3)^{\mathrm{A} \mathrm{B}}$ & $33.4(6.8)^{\mathrm{B}}$ \\
\hline
\end{tabular}

Values are means (standard deviations). $n=10$ (per group). Different superscript letters in each row indicate statistically significant differences among the storage time $(p<0.05)$. NS $=$ no significant difference $(p>0.05)$. 


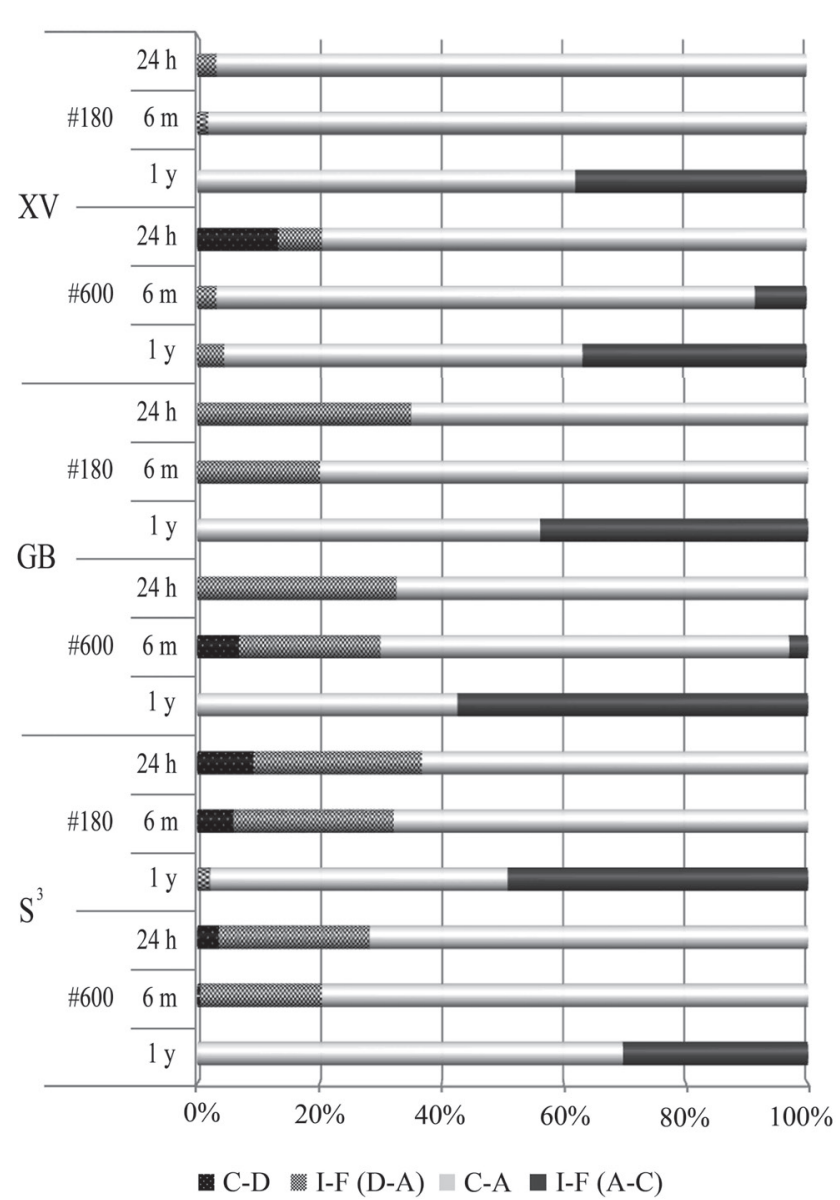

Fig. 2 Percentage distributions of failure modes of three one-step self-etch adhesives. C-D: cohesive failure in dentin, I-F (D-A): interfacial failure at dentinadhesive interface, $\mathrm{C}-\mathrm{A}$ : cohesive failure in adhesive resin, and $\mathrm{I}-\mathrm{F}(\mathrm{A}-\mathrm{C})$ : interfacial failure at adhesive-composite interface.

difference between them $(p>0.05)$. One year of water storage significantly increased the percentage of interfacial failures at adhesive-composite interface (I-F (A-C)), compared to the 24-hour control group (XV \#180; $p=0.013, \mathrm{XV} \# 600 ; p=0.013$, GB \#180; $p=0.005, \mathrm{~GB} \# 600$; $p=0.001, \mathrm{~S}^{3} \# 180 ; p=0.005$ and $\mathrm{S}^{3} \# 600 ; p=0.005$ ).

Regarding SEM observation of the de-bonded specimens, no voids were observed within the adhesive resin of each adhesive in the 24-hour control group (Figs. $3(\mathrm{a})-(\mathrm{c})$ ), while small voids were observed in the 6 -month water storage group (Figs. 3(d)-(f)). In the 6-month water storage groups of XV and GB, more and larger voids were observed within the adhesive resin in the \#180-grit SiC paper groups than in the \#600-grit $\mathrm{SiC}$ paper groups (Figs. 3(d) and 3(e)), while in the specimens of $\mathrm{S}^{3}$, there were no morphological alterations in the adhesive resin between the \#180-grit and \#600-grit SiC paper groups (Fig. 3(f)). After 1 year of water storage, all the adhesives in both the \#180-grit and \#600-grit SiC paper groups exhibited detached or missing filler particles of resin composite in the adhesive-composite interface (Figs. 3(g)-(i)).

SEM observation of the dentin surfaces treated with one-step self-etch adhesives

The conditioning effect on the dentin surfaces of the tested adhesives are shown in Fig. 4. In the \#180-grit and \#600-grit SiC paper groups of XV and GB, there were less morphological alterations in the treated dentin surfaces, in which the smear layer and smear plugs were mostly dissolved and removed (Figs. 4(a), 4(b), 4(d) and $4(\mathrm{e}))$. On the other hand, $\mathrm{S}^{3}$ was less effective in removal of the smear layer than XV and GB, in which there were remnants of the smear layer with smear plugs occluding the dentinal tubules (Figs. 4(c) and 4(f)). In addition, the \#180-grit SiC paper group exhibited more remnants of the smear layer on the treated dentin surface than the \#600-grit $\mathrm{SiC}$ paper group.

\section{Nanoleakage evaluation}

Typical observations of the resin-dentin interfaces of the 24-hour control and 1-year water storage specimens prepared with\#180- or \#600-grit SiC paper are illustrated in Figs. 5 and 6. In the specimens of XV and GB, silver uptake to a depth of 1.5 to $2.5 \mu \mathrm{m}$ was observed along the adhesive interfaces in the \#600-grit $\mathrm{SiC}$ paper groups, while in the \#180-grit $\mathrm{SiC}$ paper groups, this was observed to be from 2 to $4.5 \mu \mathrm{m}$ (Figs. 5(a), 5(b), 5(d), and $5(\mathrm{e}))$. On the other hand, the specimens of $\mathrm{S}^{3}$ in the \#180-grit and \#600-grit $\mathrm{SiC}$ paper groups did not represent distinct silver uptake at the resin-dentin interface (Figs. 5(c) and 5(f)). After 1 year of water storage, all the adhesives in both the \#180-grit and \#600-grit SiC paper groups exhibited much more silver deposition within the whole of the adhesive layer and along the adhesive-composite interface, compared to the 24-hour control specimens (Fig. 6).

\section{DISCUSSION}

The characteristics of the dentin smear layer are dependent upon the method of cutting ${ }^{26,30}$. It has been reported that dentin smear layers prepared by \#180-grit $\mathrm{SiC}$ paper are thick and rough, while those prepared with \#600-grit $\mathrm{SiC}$ paper are thin and tight ${ }^{31}$. The conditioning effect of self-etch adhesives on smear layer covered dentin surfaces are known to be influenced by their $\mathrm{pH}$ value. Generally, self-etch adhesives can be classified into three categories: mild ( $\mathrm{pH}$ of around 2.0), intermediate $(\mathrm{pH}$ of around 1.5) and strong $(\mathrm{pH}<1.0)$, depending upon the solubilizing ability of the smear layer and demineralization depth of the underlying dentin $^{32,33)}$. The $\mathrm{pH}$ values of $\mathrm{XV}$ and GB are 1.3 and 1.5, respectively, while $\mathrm{S}^{3}$ has higher a $\mathrm{pH}$ of 2.7 . In this study, there were no significant differences in the bond strengths of the 24-hour control groups between the \#180-grit and \#600-grit SiC paper groups, regardless of the $\mathrm{pH}$ values of the tested adhesives. This result is in agreement with the previous studies ${ }^{34,35)}$. They speculated 

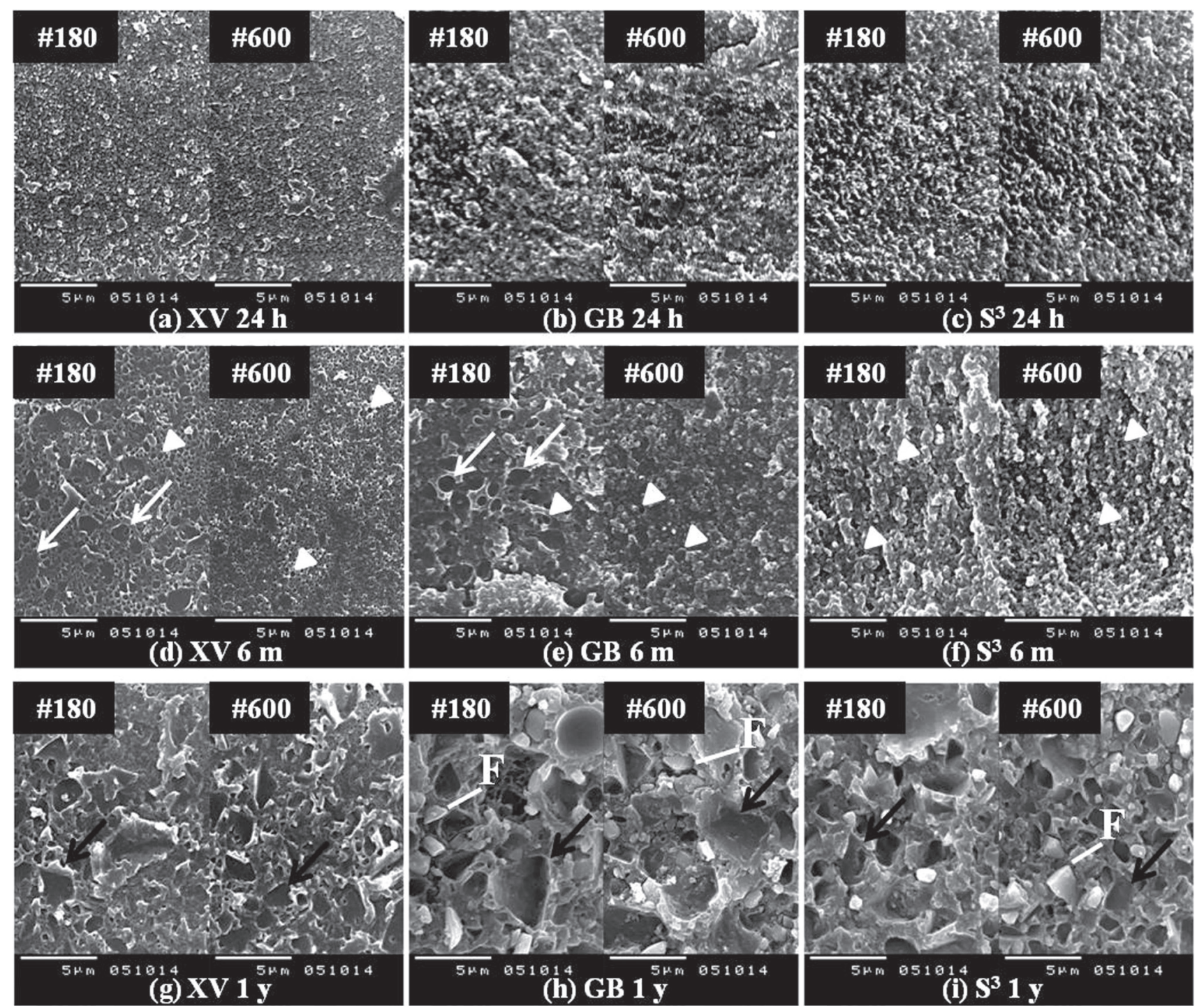

Fig. 3 Representative SEM images $(\times 5,000)$ of de-bonded specimens prepared with \#180- or \#600-grit SiC paper after 24 hours ((a)-(c)), 6 months ((d)-(f)) and 1 year ((g)-(i)) of water storage in the regions of cohesive failure in adhesive resin ((a)-(f)) and interfacial failure at the adhesive-composite interface ((g)-(i)) ((a), (d) and (g): Xeno V (XV), (b), (e) and (h): G BOND PLUS (GB), (c), (f) and (i): Clearfil $\mathrm{S}^{3}$ Bond $\left(\mathrm{S}^{3}\right)$ ). For all adhesives, small voids (arrowheads) were observed within the adhesive resin after 6 months of water storage ((d)-(f)). For XV and GB, many large voids (white arrows) were observed within the adhesive resin in the \#180-grit SiC paper groups after 6 months of water storage $((d)$ and $(e))$. For all adhesives, filler particles of resin composite were observed to be detached (F) or missing (black arrows) in the adhesive-composite interface after 1 year of water storage ((g)-(i)).

that a thick smear layer does not prevent adhesive monomer from penetrating into the underlying dentin matrix, or the buffering capacity of the smear layer is weak. In this study, the main failure mode of all 24-hour control groups was cohesive failure in adhesive resin, but not interfacial failure at dentin-adhesive interface even in the \#180-grit SiC paper group (Fig. 2). Presumably, a \#180-grit-prepared smear layer would be thicker, but more porous than a \#600-grit-prepared smear layer, allowing adhesive monomer penetration into the underlying dentin even when $\mathrm{S}^{3}$ with a higher $\mathrm{pH}$, was used.
SEM observation of the resin-dentin interface of the 24-hour control specimens revealed that the HEMA-free self-etch adhesives of XV and GB, expressed deeper silver uptake along the resin-dentin interface in the \#180-grit SiC paper groups, compared to the \#600-grit $\mathrm{SiC}$ paper groups (Figs. 5(a), 5(b), 5(d) and 5(e)). Generally, it has been demonstrated that an intermediate self-etch adhesive ( $\mathrm{pH}$ of around 1.5) can create a hybrid layer of approximately $1.5 \mu \mathrm{m}$ thick ${ }^{5}$. In this study, the depth of silver uptake of the XV and GB specimens was 1.5 to $2.5 \mu \mathrm{m}$ in the \#600-grit $\mathrm{SiC}$ paper groups, while 2 to $4.5 \mu \mathrm{m}$ in the \#180-grit $\mathrm{SiC}$ paper groups. In the case 


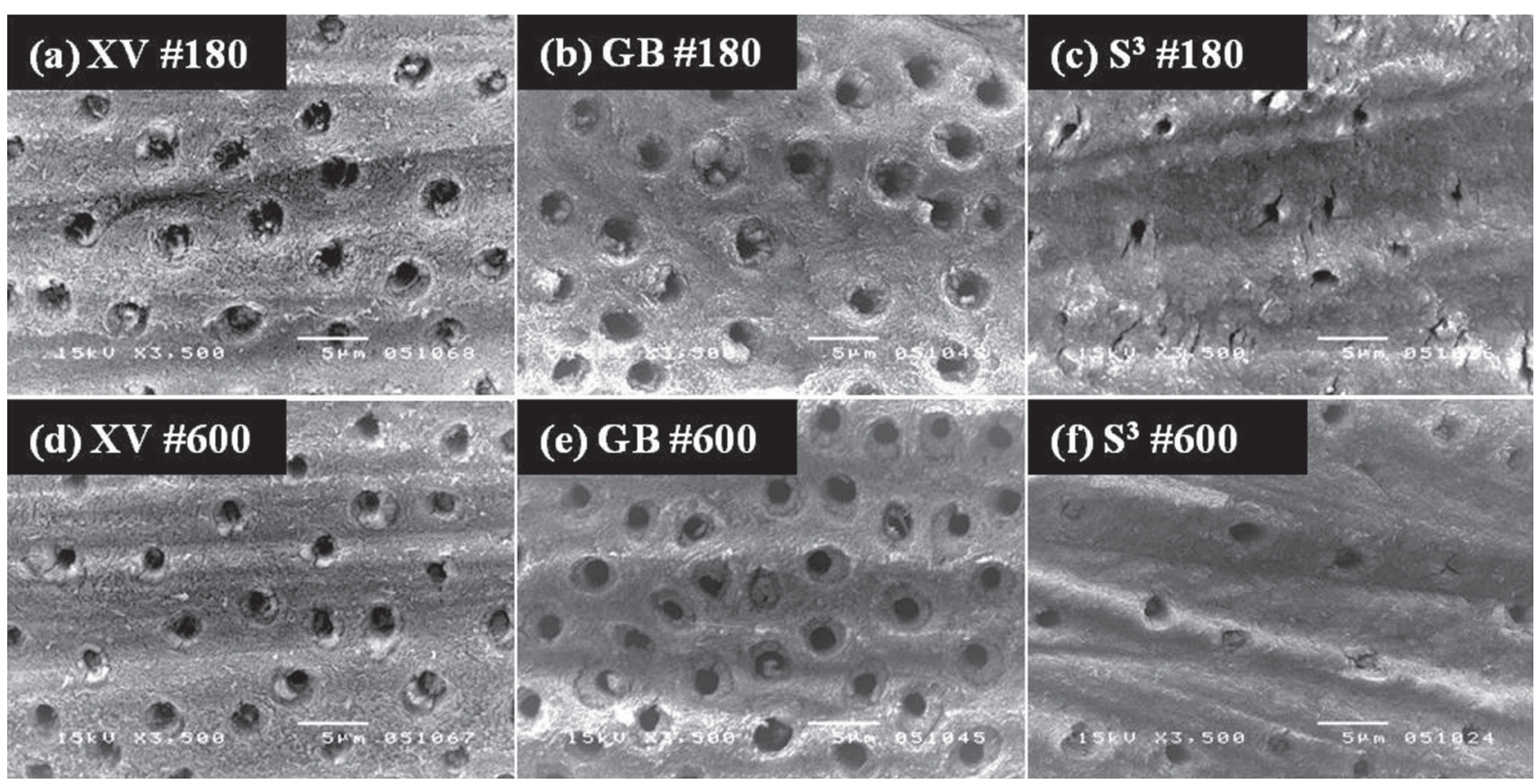

Fig. 4 Representative SEM images $(\times 3,500)$ of the dentin surfaces treated with the tested adhesives ((a) and (d): Xeno V (XV), (b) and (e): G BOND PLUS (GB), (c) and (f): Clearfil $\mathrm{S}^{3}$ Bond ( $\left.\mathrm{S}^{3}\right)$; Upper row: \#180-grit SiC paper group, lower row: \#600-grit SiC paper group). For XV and GB, the smear layer and smear plugs were mostly dissolved and removed in both the \#180-grit and \#600-grit SiC paper groups ((a), (b), (d) and (e)). For $\mathrm{S}^{3}$, the \#180-grit SiC paper group exhibited more remnants of the smear layer on the treated dentin surface than the \#600-grit SiC paper group ((c) and (f)).

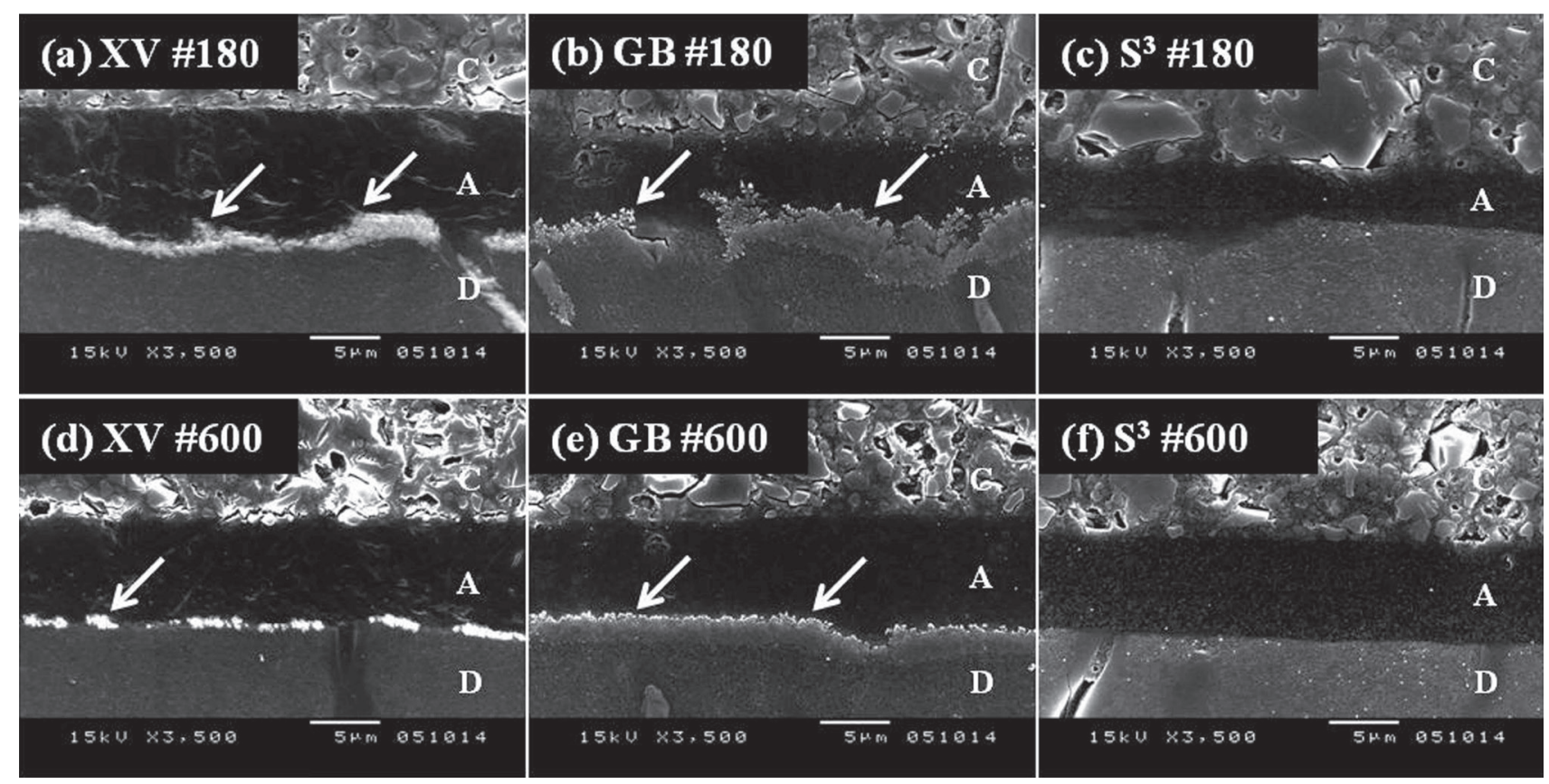

Fig. 5 Representative SEM images $(\times 3,500)$ of the resin-dentin interface of the 24-hour control specimens prepared with \#180- or \#600-grit SiC paper ((a) and (d): Xeno V (XV), (b) and (e): G BOND PLUS (GB), (c) and (f): Clearfil $\mathrm{S}^{3}$ Bond $\left(\mathrm{S}^{3}\right)$; Upper row: \#180-grit $\mathrm{SiC}$ paper group, lower row: \#600-grit $\mathrm{SiC}$ paper group). For XV and GB, silver uptake was observed within the hybrid and adhesive layer (white arrows) in both the \#180-grit and \#600-grit SiC paper groups. The depth of silver uptake was 2 to $4.5 \mu \mathrm{m}$ in the \#180-grit SiC paper groups ((a) and (b)), while 1.5 to 2.5 $\mu \mathrm{m}$ in the \#600-grit SiC paper groups ((d) and (e)). The specimens of $\mathrm{S}^{3}$ in both the \#180-grit and \#600-grit $\mathrm{SiC}$ paper groups did not represent distinct silver uptake at the resin-dentin interface ((c) and (f)). C: resin composite, A: adhesive resin, D: dentin. 


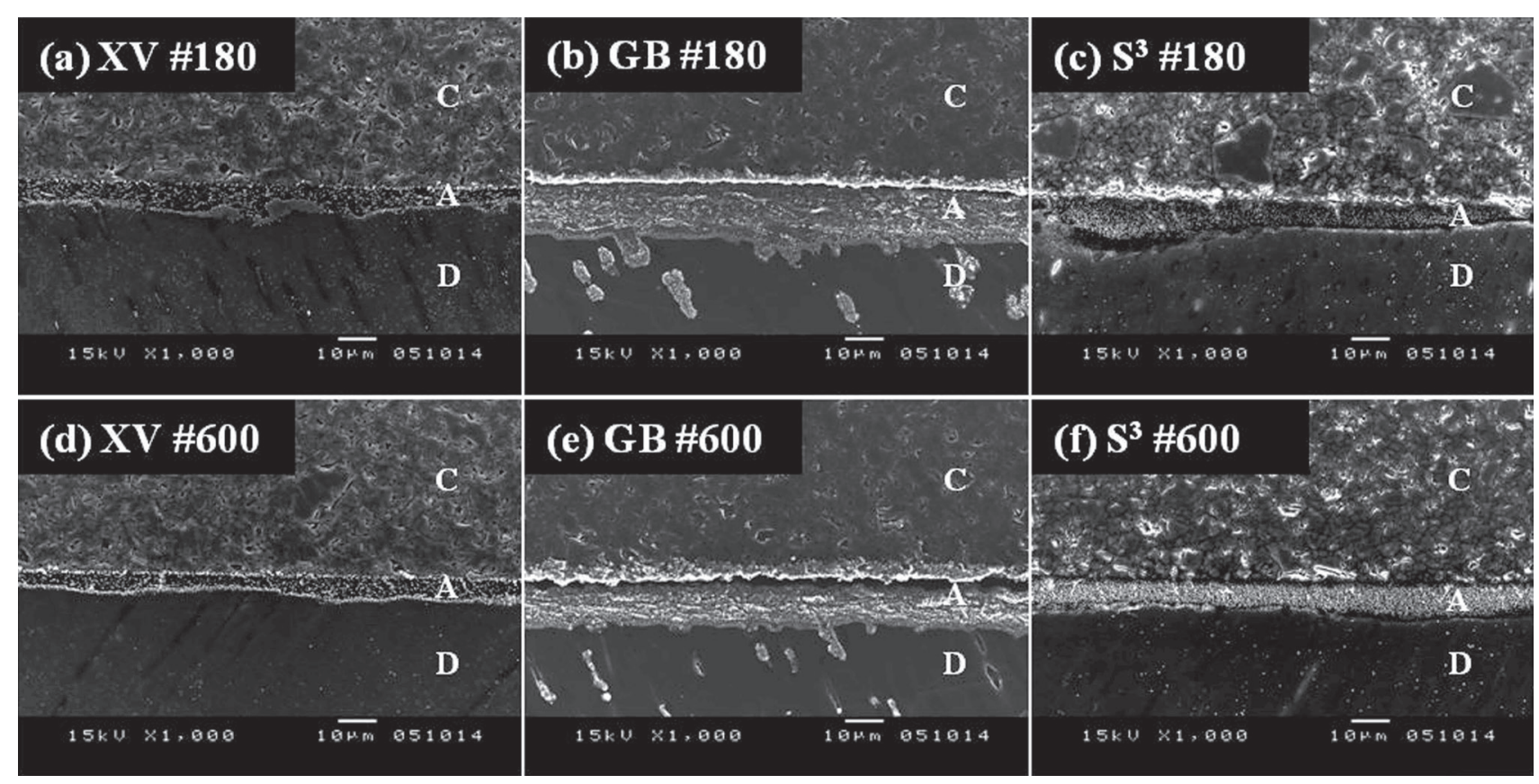

Fig. 6 Representative SEM images $(\times 1,000)$ of the resin-dentin interface of the 1-year water storage specimens prepared with \#180- or \#600-grit SiC paper ((a) and (d): Xeno V (XV), (b) and (e): G BOND PLUS (GB), (c) and (f): Clearfil S ${ }^{3}$ Bond $\left(\mathrm{S}^{3}\right)$; Upper row: \#180-grit SiC paper group, lower row: \#600-grit SiC paper group). All the adhesives in both the \#180-grit and \#600-grit SiC paper groups exhibited silver deposition within the whole of the adhesive layer and along the adhesive-composite interface. C: resin composite, A: adhesive resin, D: dentin.

of HEMA-free self-etch adhesives, water-tree nanoleakage with nanoleakage within the hybrid layer has often been observed, which is caused by the water movement across the resin-dentin interface from dentinal tubules, located along the interface and extending into the adhesive layer during the bonding procedure ${ }^{21,22,29)}$. Therefore, the silver uptake observed in this study, would represent both nanoleakage within the hybrid layer and water-tree nanoleakage. XV $(\mathrm{pH}=1.3)$ and $\mathrm{GB}(\mathrm{pH}=1.5)$ could dissolve and remove the \#600-gritprepared and \#180-grit-prepared smear layer (Figs. 4(a), 4(b), 4(d) and 4(e)). On the other hand, the \#180-gritprepared smear layer may include a larger amount of water than the \#600-grit-prepared smear layer, because the \#180-grit-prepared smear layer is thicker and more porous. In addition, the porous structure of the \#180-gritprepared smear layer would not prevent water diffusion from dentinal tubules even though it is thick. Therefore, the adhesives in the \#180-grit SiC paper group might suffer from water-contamination during the bonding procedure. As a result, it is speculated that XV and GB exhibited more water-tree nanoleakage formation in the \#180-grit SiC paper group more than in the \#600-grit $\mathrm{SiC}$ paper group. Water-trees might act as initiation sites for hydrolytic degradation of the adhesive resin, allowing water diffusion across the adhesive layer from dentinal tubules ${ }^{17,22)}$.

On the other hand, the HEMA-containing adhesive, $\mathrm{S}^{3}$, did not exhibit silver uptake at the resin-dentin interface in either the \#180-grit or \#600-grit SiC paper groups (Figs. 5(c) and 5(f)). These results would indicate that $\mathrm{S}^{3}$ expresses less nanoleakage within the hybrid layer as well as water-tree nanoleakage. This result is in agreement with the previous study using a $\mathrm{TEM}^{36)}$. HEMA can improve the bond strength to dentin, enhancing wetting of the dentin subsurface ${ }^{37)}$, and preventing phase separation between the hydrophobic components and water ${ }^{23)}$, resulting in the absence of water-tree nanoleakage formation. However, HEMA promotes poly-HEMA hydrogels with residual water on the adhesive interface and/or diffused water from dentinal tubules. These water-forming poly-HEMA hydrogels within the adhesive resin permit water movement across the adhesive layer, leading to hydrolytic degradation of the adhesive resin ${ }^{12,38)}$.

After XV and GB were stored for 6 months in water storage, the \#180-grit $\mathrm{SiC}$ paper group showed significantly lower bond strength than the \#600-grit SiC paper group. Regarding the failure modes, both surface preparation groups displayed mainly cohesive failure in adhesive resin, in which there were no significant differences between them (Fig. 2). However, SEM observation of the fractured surfaces of XV and GB revealed many large voids within the adhesive resin in the \#180-grit $\mathrm{SiC}$ paper groups with less voids in the \#600-grit SiC paper groups (Figs. 3(d) and 3(e)). These results might indicate that in the \#180-grit SiC paper groups of XV and GB, larger amounts of water-tree nanoleakage caused degradation of the adhesive resin, resulting in lower bond strengths than the \#600-grit $\mathrm{SiC}$ 
paper groups. On the other hand, $\mathrm{S}^{3}$ showed no significant difference in $\mu$ TBS and failure mode between the \#180-grit and \#600-grit SiC paper groups after 6 months of water storage (Fig. 2), and there were also no morphological alterations in the adhesive layer of the fractured surfaces between them (Fig. 3(f)). These results might indicate that for $\mathrm{S}^{3}$, smear layer characteristics did not affect bond strength to dentin during 6-month water storage.

One year of water storage significantly decreased bond strengths of all the tested adhesives in both the \#180-grit and \#600-grit $\mathrm{SiC}$ paper groups. For each adhesive, there was no significant difference in bond strength between the surface preparation groups. In addition, SEM observation of resin-dentin interface revealed that all the 1-year water storage specimens exhibited much more silver deposition within the whole adhesive layer than the 24-hour control specimens (Fig. 6 ). These results would indicate hydrolytic degradation of the adhesive resin. On the other hand, in the failure mode analysis, all the adhesives exhibited significantly increased percentages of failure at the adhesivecomposite interface after 1 year of water storage, regardless of the dentin surface preparation method (Fig. 2). These results would indicate that the adhesivecomposite interface was weaker than the adhesive layer. The join of this interface is completed by co-polymerization between hydrophobic resin matrix of resin composite and the uncured layer (the oxygen inhibition layer) at the top of adhesive layer. In the case of one-step self-etch adhesives, residual water/solvents might be present within the uncured layer, which would impair co-polymerization between the hydrophilic uncured resin and hydrophobic resin composite, because the presence of residual water/solvents causes a reduction in the degree of conversion ${ }^{13,15,39)}$. Moreover, after long-term water storage, diffused water across the adhesive layer would be trapped as water droplets underneath the hydrophobic resin composite accumulate along the adhesive-composites interface ${ }^{40)}$. For one-step self-etch adhesives, the adhesive-composite interface may be the most vulnerable region after 1 year of water storage, regardless of the presence of HEMA in the adhesives and/or smear layer characteristics.

In this study, sectioned slabs were stored in water for 6 months or 1 year. When compared to the storage of whole teeth in water, the narrow diameter of the sectioned slabs can accelerate the speed at which water can enter the resin-dentin interface ${ }^{9}$. Thus, the results of this study showed the significant decrease in $\mu$ TBS of all the adhesives after 1 year of water storage and the morphological degradation of resin-dentin interface. However, it is not unclear that this bond degradation would be seen in vivo. Further in vivo long-term studies are required to evaluate the dentin bonding durability of HEMA-free or HEMA-containing adhesives.

Within the limitations of this study, it was concluded that for the HEMA-free self-etch adhesives of XV and GB, the smear layer characteristics affected water-tree nanoleakage formation in the adhesive layer, which contributed to reduced dentin bond strengths after 6 months of water storage, while the characteristics did not affect bond strength of the HEMA-containing adhesive, $S^{3}$. However, after 1 year of water storage, the bond strengths of all the tested adhesives were not influenced by the smear layer characteristics or the presence of HEMA in the adhesives, with increasing failure at the adhesive-composite interface.

\section{ACKNOWLEDGMENTS}

This work was supported by the grant from the Japanese Ministry of Education, Global Center of Excellence (GCOE) Program, "International Research Center for Molecular Science in Tooth and Bone Diseases".

\section{REFERENCES}

1) Sano H, Takatsu T, Ciucchi B, Horner JA, Matthews WG, Pashley DH. Nanoleakage: leakage within the hybrid layer. Oper Dent 1995; 20: 18-25.

2) Tay FR, Hashimoto M, Pashley DH, Peters MC, Lai SC, Yiu CK, Cheong C. Aging affects two modes of nanoleakage expression in bonded dentin. J Dent Res 2003; 82: 537-541.

3) De Munck J, Van Meerbeek B, Yoshida Y, Inoue S, Vargas M, Suzuki K, Lambrechts P, Vanherle G. Four-year water degradation of total-etch adhesives bonded to dentine. J Dent Res 2003; 82: 136-140.

4) Okuda M, Pereira PNR, Nakajima M, Tagami J, Pashley DH. Long-term durability of resin-dentine interface: nanoleakage vs. microtensile bond strength. Oper Dent 2002; 27: 289-296.

5) Van Landuyt KL, Mine A, De Munck J, Jaecques S, Peumans M, Lambrechts P, Van Meerbeek B. Are one-step adhesives easier to use and better performing? Multifactorial assessment of contemporary one-step self-etching adhesives. J Adhes Dent 2009; 11: 175-190.

6) Margvelashvili M, Goracci C, Beloica M, Papacchini F, Ferrari M. In vitro evaluation of bonding effectiveness to dentin of all-in-one adhesives. J Dent 2010; 38: 106-112.

7) Hashimoto M, Fujita S, Kaga M, Yawaka Y. In vitro durability of one-bottle resin adhesives bonded to dentin. Dent Mater J 2007; 26: 677-686.

8) Torkabadi S, Nakajima M, Ikeda M, Foxton RM, Tagami J. Bonding durability of HEMA-free and HEMA-containing one-step adhesives to dentine surrounded by bonded enamel. J Dent 2008; 36: 80-86.

9) Foxton RM, Melo L, Stone DG, Pilecki P, Sherriff M, Watson TF. Long-term durability of one-step adhesive-composite systems to enamel and dentin. Oper Dent 2008; 33: 651-657.

10) Itoh S, Nakajima M, Hosaka K, Okuma M, Takahashi M, Shinoda Y, Seki N, Ikeda M, Kishikawa R, Foxton RM, Tagami J. Dentin bond durability and water sorption/ solubility of one-step self-etch adhesives. Dent Mater J 2010; 29: 623-630.

11) Ito S, Hashimoto M, Wadgaonkar B, Svizero N, Carvalho RM, Yiu C, Rueggeberg FA, Foulger S, Saito T, Nishitani Y, Yoshiyama M, Tay FR, Pashley DH. Effects of resin hydrophilicity on water sorption and changes in modulus of elasticity. Biomaterials 2005; 26: 6449-6459.

12) Yiu CK, Pashley EL, Hiraishi N, King NM, Goracci C, Ferrari M, Carvalho RM, Pashley DH, Tay FR. Solvent and water retention in dental adhesive blends after evaporation. Biomaterials 2005; 26: 6863-6872.

13) Malacarne J, Carvalho RM, de Goes MF, Svizero N, Pashley DH, Tay FR, Yiu CK, Carrilho MR. Water sorption/solubility of dental adhesive resins. Dent Mater 2006; 22: 973-980. 
14) Nishitani Y, Yoshiyama M, Hosaka K, Tagami J, Donnelly A, Carrilho M, Tay FR, Pashley DH. Use of Hoy's solubility parameters to predict water sorption/solubility of experimental primers and adhesives. Eur J Oral Sci 2007; 115: 81-86.

15) Tay FR, Pashley DH. Have dentin adhesives become too hydrophilic? J Can Dent Assoc 2003; 69: 726-731.

16) Tay FR, Pashley DH, Suh B, Carvalho R, Miller M. Single-step, self-etch adhesives behave as permeable membranes after polymerization. Part I. Bond strength and morphologic evidence. Am J Dent 2004; 17: 271-278.

17) Tay FR, Pashley DH, Garcìa-Godoy F, Yiu CK. Single-step, self-etch adhesives behave as permeable membranes after polymerization. Part II. Silver tracer penetration evidence. Am J Dent 2004; 17: 315-322.

18) Hosaka K, Tagami J, Nishitani Y, Yoshiyama M, Carrilho M, Tay FR, Agee KA, Pashley DH. Effect of wet vs. dry testing on the mechanical properties of hydrophilic self-etching primer. Eur J Oral Sci 2007; 115: 239-245.

19) Hosaka K, Nakajima M, Takahashi M, Itoh S, Ikeda M, Tagami J, Pashley DH. Relationship between mechanical properties of one-step self-etch adhesives and water sorption. Dent Mater 2010; 26: 360-367.

20) Yuan Y, Shimada Y, Ichinose S, Tagami J. Qualitative analysis of adhesive interface nanoleakage using FE-SEM/ EDS. Dent Mater 2007; 23: 561-569.

21) Hashimoto M, Fujita S, Kaga M, Yawaka Y. Effect of water on bonding of one-bottle self-etching adhesives. Dent Mater J 2008; 27: 172-178.

22) Tay FR, Pashley DH, Suh BI, Hiraishi N, Yiu CK. Water treeing in simplified dentin adhesives--déjà vu? Oper Dent 2005; 30: 561-579.

23) Van Landuyt KL, De Munck J, Snauwaert J, Coutinho E, Poitevin A, Yoshida Y, Inoue E, Peumans M, Suzuki K, Lambrechts P, Van Meerbeek B. Monomer-solvent phase separation in one-step self-etching adhesives. J Dent Res 2005; 84: 183-188.

24) Tay FR, Sano H, Carvalho R, Pashley EL, Pashley DH. An ultrastructural study of the influence of acidity of self-etching primers and smear layer thickness on bonding to intact dentin. J Adhes Dent 2000; 2: 83-98.

25) Tay FR, Carvalho R, Sano H, Pashley DH. Effect of smear layers on the bonding of a self-etching primer to dentin. $J$ Adhes Dent 2000; 2: 99-116.

26) Oliveira SS, Pugach MK, Hilton JF, Watanabe LG, Marshall SJ, Marshall GW Jr. The influence of the dentin smear layer on adhesion: a self-etching primer vs. a total-etch system. Dent Mater 2003; 19: 758-767.

27) Yoshida E, Uno S. Voids formation along the bonding interface between a smeared dentin surface and all-in-one adhesives. Dent Mater J 2004; 23: 643-649.

28) Perdigao J, Lambrechts P, Van Meerbeek B, Vanherle G, Lopes AL. Field emission SEM comparison of four postfixation drying techniques for human dentin. J Biomed Mater Res 1995; 29: 1111-1120.

29) Tay FR, Pashley DH, Yoshiyama M. Two modes of nanoleakage expression in single-step adhesives. J Dent Res 2002; 81: 472-476.

30) Barros JA, Myaki SI, Nör JE, Peters MC. Effect of bur type and conditioning on the surface and interface of dentine. $J$ Oral Rehabil 2005; 32:849-856.

31) Watanabe I, Saimi Y, Nakabayashi N. Effect of smear layer on bonding to ground dentin. Relationship between grinding conditions and tensile bond strength. J Jpn Dent Mater 1994; 13: 101-108.

32) De Munck J, Vargas M, Iracki J, Van Landuyt K, Poitevin A, Lambrechts P, Van Meerbeek B. One-day bonding effectiveness of new self-etch adhesives to bur-cut enamel and dentin. Oper Dent 2005; 30: 39-49.

33) De Munck J, Van Landuyt K, Peumans M, Poitevin A, Lambrechts P, Braem M, Van Meerbeek B. A critical review of the durability of adhesion to tooth tissue: methods and results. J Dent Res 2005; 84: 118-132.

34) Tani C, Finger WJ. Effect of smear layer thickness on bond strength mediated by three all-in-one self-etching priming adhesives. J Adhes Dent 2002; 4: 283-289.

35) Reis A, Grandi V, Carlotto L, Bortoli G, Patzlaff R, Rodrigues Accorinte Mde L, Dourado Loguercio A. Effect of smear layer thickness and acidity of self-etching solutions on early and long-term bond strength to dentin. J Dent 2005; 33: 549-559.

36) Yuan Y, Shimada Y, Ichinose S, Sadr A, Tagami J. Effects of dentin characteristics on interfacial nanoleakage. J Dent Res 2007; 86: 1001-1006

37) Van Meerbeek B, De Munck J, Yoshida Y, Inoue S, Vargas M, Vijay P, Van Landuyt K, Lambrechts P, Vanherle G. Buonocore memorial lecture. Adhesion to enamel and dentin: current status and future challenges. Oper Dent 2003; 28: 215-235

38) Yiu CK, King NM, Pashley DH, Suh BI, Carvalho RM, Carrilho MR, Tay FR. Effect of resin hydrophilicity and water storage on resin strength. Biomaterials 2004; 25: 5789-5796.

39) Cadenaro M, Antoniolli F, Sauro S, Tay FR, Di Lenarda R, Prati C, Biasotto M, Contardo L, Breschi L. Degree of conversion and permeability of dental adhesives. Eur J Oral Sci 2005; 113: 525-530.

40) Hashimoto M, Fujita S, Endo K, Ohno H. In vitro degradation of resin-dentin bonds with one-bottle self-etching adhesives. Eur J Oral Sci 2009; 117: 611-617. 